\title{
La flore des systèmes agropastoraux de la Basse Casamance (Sénégal) : cas de la communauté rurale de Mlomp
}

\author{
César BASSENE ${ }^{1 *}$, Mame Samba MBAYE ${ }^{2}$, Abdou Aziz CAMARA ${ }^{2}$, \\ Aboubakry KANE ${ }^{2}$, Madiop GUEYE², Samba Ndao SYLLA², \\ Bienvenu $\mathrm{SAMBOU}^{3}$ et Kandioura NOBA ${ }^{2}$ \\ ${ }^{I}$ Section Productions Végétales et Agronomie, UFR des Sciences Agronomiques, de l'Aquaculture \\ et des Technologies Alimentaires, Université Gaston Berger de Saint Louis, BP. 234 Saint Louis. \\ ${ }^{2}$ Laboratoire de Botanique et Biodiversité, Département de Biologie Végétale, \\ Faculté des Sciences et Techniques, Université Cheikh Anta Diop, Sénégal. \\ ${ }^{3}$ Institut des Sciences de l'Environnement, Faculté des Sciences et Techniques, \\ Université Cheikh Anta Diop, Sénégal. \\ *Auteur correspondant ; E-mail: cesar.bassene@yahoo.fr; Tel : (221) 775567279
}

\section{RESUMĒ}

Ce travail constitue une première étude de la flore des systèmes agropastoraux dans la communauté rurale de Mlomp. Cette étude est réalisée par la méthode des relevés phytosociologiques. Elle a été entreprise pour déterminer la structure de la flore (spectres taxonomique, biologique et chorologique) et l'influence du système d'exploitation des terres sur la flore. Les résultats indiquent que cette flore globale est constituée de 158 espèces réparties dans 91 genres et 37 familles. Dans cette flore, 6 Familles principales regroupent plus de $3 / 5(62,66 \%)$ des espèces: les Poaceae (26,6\%), les Fabaceae (12,02\%), les Cyperaceae (6,96\%), les Malvaceae $(6,96 \%)$, les Convolvulaceae $(5,06 \%)$ et les Rubiaceae $(5,06 \%)$. Cette flore est caractérisée par une prédominance des thérophytes $(77,21 \%)$ avec toutefois la présence de phanérophytes $(9,49 \%)$, d'hémicrytophytes $(7,6 \%)$, de géophytes $(4,43 \%)$ et de chaméphytes $(1,27 \%)$. Cette flore est composée, pour l'essentiel, d'espèces africaines $(53,2 \%)$, pantropicales $(26,58 \%)$ et d'espèces afro-asiatiques $(6,96 \%)$. La flore des pâturages est plus diversifiée que celle des écosystèmes cultivés. Par contre, le système d'exploitation n'influe pas fortement sur les spectres biologique et chorologique.

(c) 2014 International Formulae Group. All rights reserved.

Mots clés : Flore, système agropastoral, champs, pâturages, Mlomp.

\section{INTRODUCTION}

L'agriculture joue un rôle important dans l'économie des pays en développement et constitue la principale source de nourriture, de revenu et d'emploi pour leurs populations rurales (Bassène, 2014). Cette agriculture constitue un secteur clé des économies ouestafricaines, assurant 30 à 50\% du PIB de la plupart des pays et représentant la plus grande source de revenus et de moyens d'existence pour 70 à $80 \%$ de la population (Toulmin et Guèye, 2003).

Au Sénégal, l'agriculture occupe près de $70 \%$ de la population (Noba, 2004, Mbaye, 2013). Au sud du pays, dans la Basse Casamance, l'agriculture constitue l'une des 
principales activités de production et est essentiellement pluviale et saisonnière (Bassène, 2008). La plupart des activités agricoles sont organisées et mises en œuvre par les exploitations familiales. Au sein de l'exploitation familiale, l'accès aux terres et aux biens agricoles s'acquiert généralement par héritage ou autres arrangements sociaux, tels que des prêts. Ces exploitations familiales se caractérisent en outre par un ensemble d'activités impliquant divers productions végétales et animales, la pêche, la chasse et la cueillette (Zoundi et Léonidas, 2003).

Cette zone sud du Sénégal est considérée comme une zone de haute concentration de la biodiversité végétale (Bâ et Noba, 2001). La dégradation des ressources végétales et la perte de la biodiversité sont des faits constatés au Sénégal comme dans les pays sahéliens depuis quelques décennies. Les causes sont liées à des facteurs divers d'ordre Climatiques, économiques et socioculturels. La sauvegarde de la biodiversité dans ces zones passe essentiellement par une connaissance de la flore et de la végétation, une connaissance des conditions écologiques des espèces rares et menacées, une appréciation de besoins énergétiques, alimentaires et médicaux des populations (Bassène, 2008). C'est dans cette optique que nous avions choisi comme première étude dans la communauté rurale de Mlomp, d'inventorier les systèmes agropastoraux.

Le présent travail envisage d'étudier la flore des systèmes agropastoraux de la communauté rurale de Mlomp et se propose, d'établir les spectres taxonomique et biologique, d'indiquer la répartition géographique des espèces inventoriées et d'analyser l'influence du système d'exploitation terres sur la flore.

\section{MATERIEL ET METHODES}

\section{Présentation de la zone d'étude}

L'étude a été réalisée dans la partie sud-ouest du Sénégal en 2008, dans les systèmes agropastoraux de la communauté rurale de Mlomp du Département d'Oussouye de la région de Ziguinchor. Cette communauté rurale de Mlomp se situe sur la rive gauche du fleuve Casamance entre $12^{\circ} 28$ et $12^{\circ} 39$ de latitude nord et $16^{\circ} 29$ et $16^{\circ} 41$ de longitude ouest. Elle est limitée à l'est par le Kameubeul bolong et à l'ouest par la communauté rurale de Diembéring. Au nord, elle est limitée par le fleuve Casamance et au sud par la communauté rurale d'Oukout (Figure 1).

\section{Inventaire floristique}

Le travail a consisté à faire des inventaires floristiques dans ces systèmes agropastoraux. Les inventaires floristiques ont démarré le mois d'août et se sont poursuivis durant toute la saison des pluies jusqu'au mois de novembre. Nous avons choisi la méthode des relevés phytosociologiques de Vanden Berghen (1982). Cette méthode consiste à délimiter une aire minimale pour chaque surface de relevé avant de délimiter l'aire du relevé qui est supérieure ou égale à l'aire minimale.

\section{Identification et nomenclature des espèces}

Les identifications des espèces ont été effectuées à l'aide :

- des Flores (Hutchinson et al., 1958 ; Merlier et Montegut, 1982 ; Le Bourgeois et Merlier, 1995) ;

- des travaux de notre laboratoire (Noba et al., 2004 ; Sarr et al., 2007) ;

- d'échantillons de l'herbier du Département de Biologie Végétale de la Faculté de Sciences et Techniques de l'Université Cheikh Anta Diop de Dakar.

La nomenclature employée est celle de Lebrun et Stork (1991).

Détermination des types biologiques et la répartition géographiques des espèces

Les types biologiques ont été déterminés à partir de la classification de Raunkier (1934) adaptée à la zone tropicale (Trochain, 1966 ; Lebrun, 1966), qui distingue les Phanérophytes (P), les Chaméphytes (C), les Hémicrytophytes $(\mathrm{H})$ et les Thérophytes (T).

Pour la répartition géographique, les informations proviennent des travaux de Traoré et Maillet (1992) et des travaux de Poilecot (1999). 

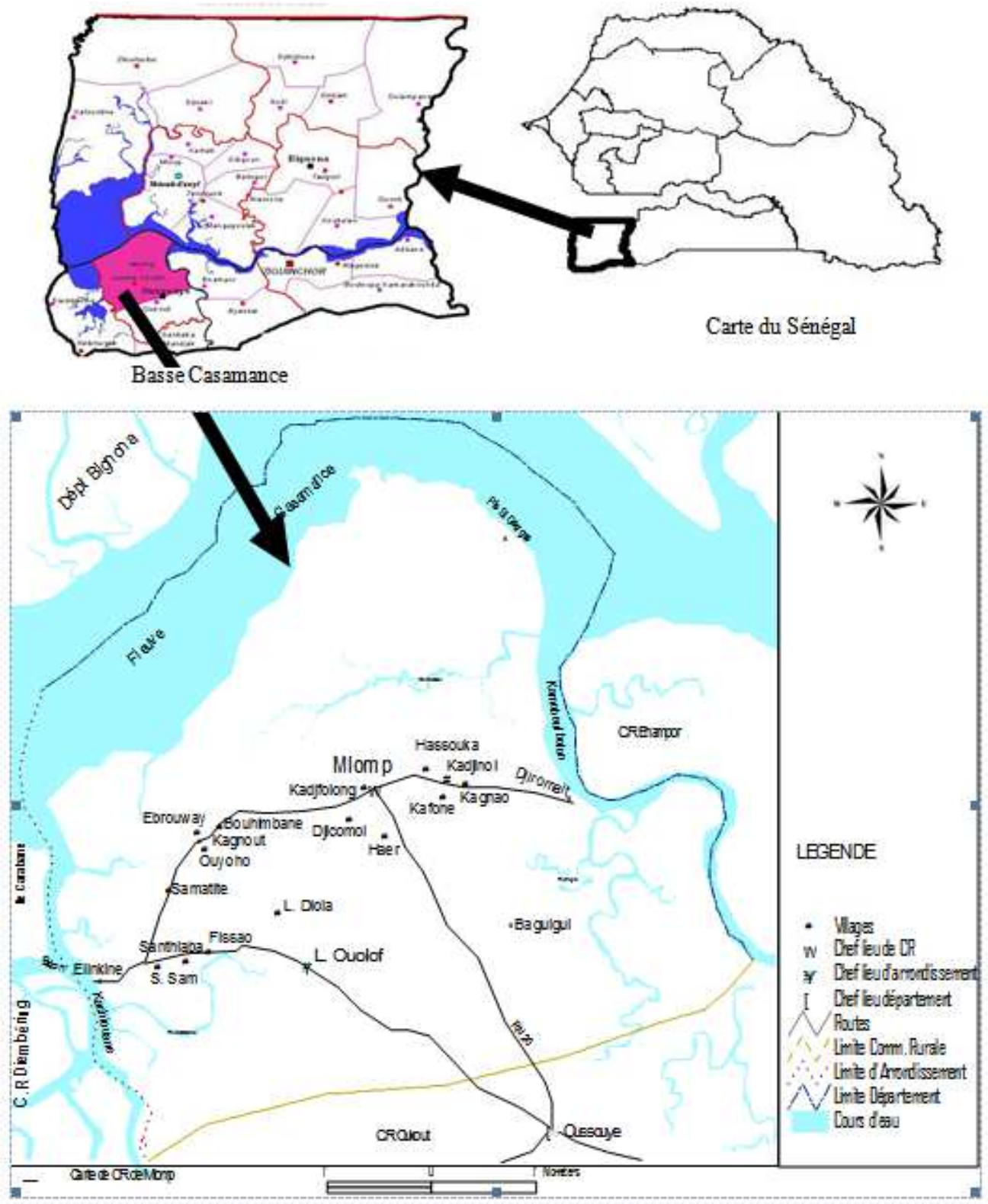

Figure 1 : Carte de la communauté rurale de Mlomp (Bassène, 2008). 


\section{RESULTATS}

Analyse de la flore globale Spectre taxonomique

Le Tableau 1 représente la liste des espèces recensées dans les systèmes agropastoraux de la Communauté rurale de Mlomp. Chaque espèce est définie par sa présence dans la zone des pâturages et les champs, son type biologique et sa répartition géographique.

Au total, 158 espèces ont été recensées dans les systèmes agropastoraux de la communauté rurale de Mlomp, appartenant à 91 genres répartis dans 37 familles.

Le Tableau 2 représente la répartition par famille des espèces de la flore globale des systèmes agropastoraux et de celle de la zone des champs et des pâturages de Mlomp.

Les résultats de ce tableau révèlent que les Dicotylédones représentent 63,29\% des espèces et les Monocotylédones 36,71\%. Les Monocotylédones, représentées principalement par les Poaceae et les Cyperaceae, représentent les familles dominantes dans les systèmes agropastoraux. Ces résultats montrent aussi que les familles plurispécifiques (avec un nombre d'espèces supérieur ou égal à 2) représentent $48,65 \%$ des 37 familles de la flore globale contre $51,35 \%$ des familles monospécifiques de la flore globale.

La Figure 2 donne des indications sur la structure de la flore des systèmes agropastoraux. Les résultats de cette figure montrent que le nombre de Dicotylédones dans les différents taxa est supérieur à celui des Monocotylédones dans toutes les flores (globale, pâturages et champs). Ces résultats révèlent aussi que le nombre de Dicotylédones des taxa dans les pâturages est proche de celui des Dicotylédones dans la flore globale contrairement dans les champs.

\section{Spectre biologique}

La Figure 3 montre les types biologiques des espèces recensées dans les systèmes agropastoraux. La flore des systèmes agropastoraux de Mlomp se rattachent à 5 types biologiques. Elle est caractérisée par une forte dominance des thérophytes $(77,21 \%)$.

\section{Spectre chorologique}

La Figure 4 représente la répartition des espèces des systèmes agropastoraux de Mlomp, regroupées en fonction de leurs affinités biogéographiques. La flore globale est largement dominée par les espèces africaines et pantropicales qui représentent ensemble près de $80 \%$ des espèces. En effet, les espèces africaines représentent plus de la moitié des espèces $(53,2 \%)$ tandis que les espèces pantropicales comptent plus du $1 / 4$ des espèces $(26,58 \%)$ de cette flore.

\section{Influence du systeme d'exploitation des terres \\ Influence du système d'exploitation des terres sur la structure de la flore \\ Le Tableau 2 indique aussi une} comparaison de la répartition en nombre espèces par famille dans les champs et les pâturages. L'analyse du Tableau 2 montre que les pâturages sont plus diversifiés que les champs. Les pâturages totalisent 147 espèces réunies dans 87 genres et 36 familles contre 102 espèces réparties dans 59 genres et 21 familles pour les champs.

Les résultats de ce tableau montrent que 23 familles sont communes pour les deux zones. 14 familles sont uniquement retrouvées dans une des zones. Dans les familles communes, 10 familles représentent le même nombre d'espèces et 13 familles représentent de différents nombre d'espèces avec 12 familles plus spécifiées dans les pâturages contre une famille (Asteraceae) plus spécifiées dans les champs. Parmi les familles communes, seule la famille des Poaceae présentent une différence importante de 15 espèces. Enfin, pour les familles uniques à une zone, 13 familles sont spécifiques aux pâturages contre une famille spécifique aux champs (Onagraceae : Ludwigia abyssinica).

Ces résultats révèlent aussi que sur les 91 genres de la flore globale, certains genres sont communs et d'autres sont uniquement répertoriés dans une zone. La majeure partie des genres sont communs avec 53 genres sur 91 contre 39 spécifiques à une zone. Dans cette spécificité, 34 genres sont répertoriés 
uniquement dans les pâturages et 5 autres genres dans les champs tels que le genre Blumea, Vernonia, Vigna, paspalum.

Influence du système d'exploitation sur les types biologiques des espèces

Le Tableau 3 montre les types biologiques des espèces recensées dans les champs et pâturages. L'analyse des résultats de ce tableau révèle que les types biologiques des espèces recensées des deux zones sont largement dominés par les thérophytes avec $82,35 \%$ des types biologiques des espèces recensées dans les champs et $76,87 \%$ des types biologiques des espèces répertoriées dans les pâturages. Ces thérophytes sont suivis des hémicryptophyes avec $6,86 \%$ et des phanérophytes avec 5, 88\% dans les champs alors que dans les pâturages ils sont suivis des phanérophytes avec $10,20 \%$ et des hémicryptophytes avec $6,80 \%$.

Influence du système d'exploitation sur la répartition géographique des espèces

Le Tableau 4 représente la répartition des espèces dans les champs et les pâturages regroupées en fonction de leurs affinités biogéographiques. Les résultats de ce tableau montrent que dans les deux zones, les espèces africaines et les espèces pantropicales dominent les flores avec plus de $80 \%$ dans chaque zone et les $20 \%$ restant sont représentées par les espèces des autres régions. Ces résultats révèlent aussi l'absence des espèces Afro-malgaches asiatiques et australiennes (Masu) et les espèces afromalgaches et américaines (Mam) dans les champs et les espèces américaines $(\mathbf{M})$ dans les pâturages.

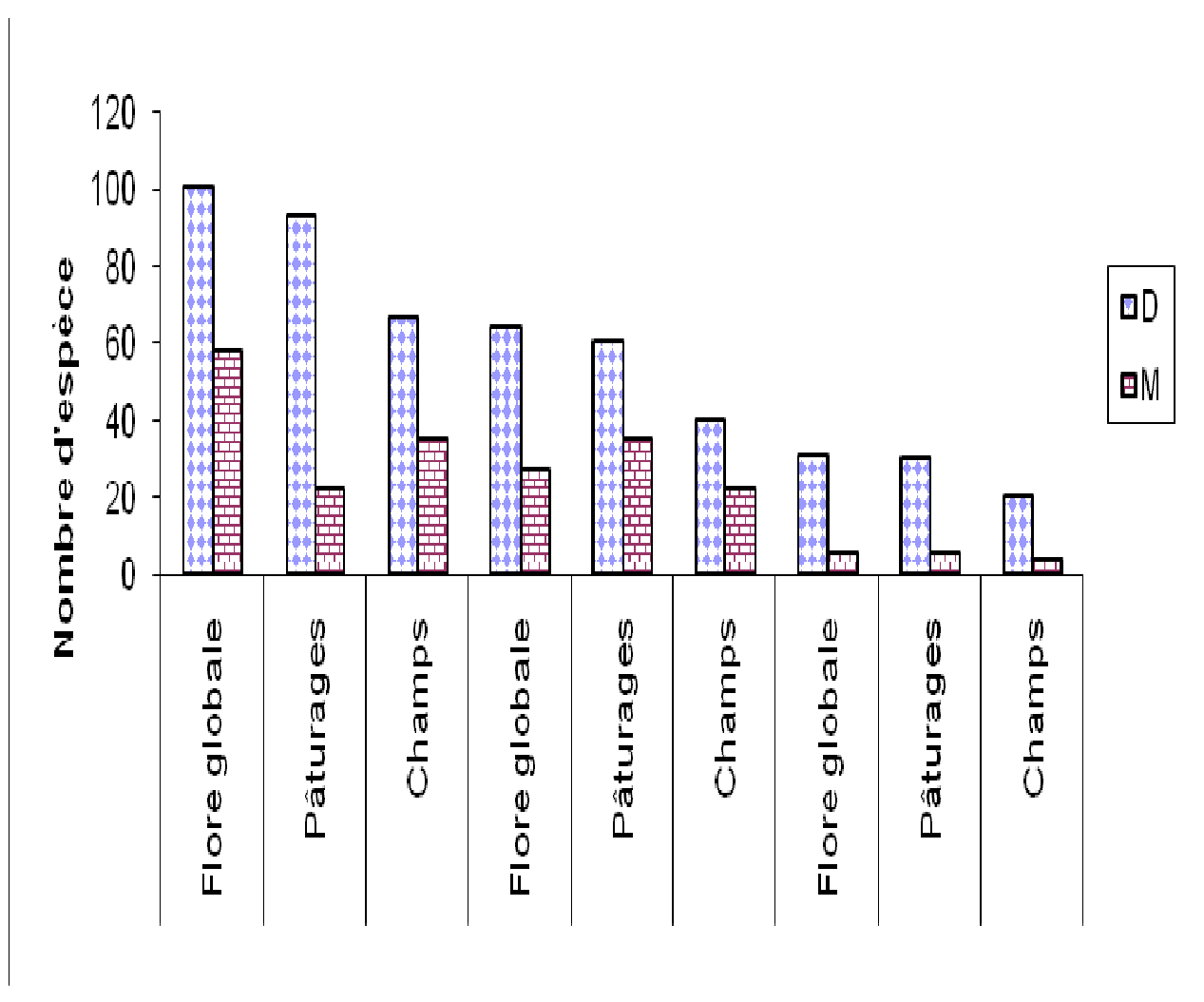

Figure 2: Structure de la flore des systèmes agropastoraux. 


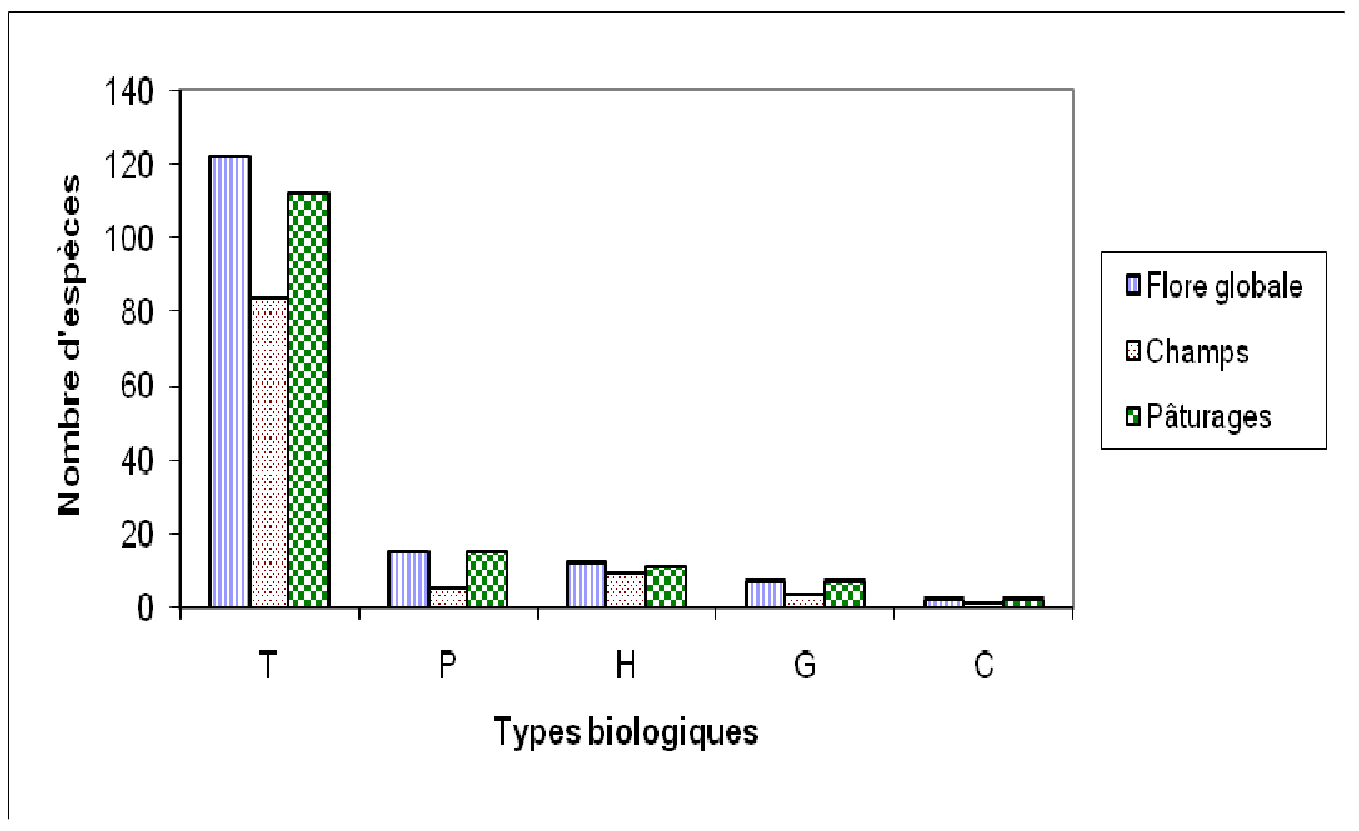

Figure 3 : Types biologiques des espèces dans les systèmes agropastoraux de Mlomp. Thérophytes (T) ; Hémicryptophytes (H) ; Géophytes (G); Chaméphytes (C) ; Phanérophytes (P).

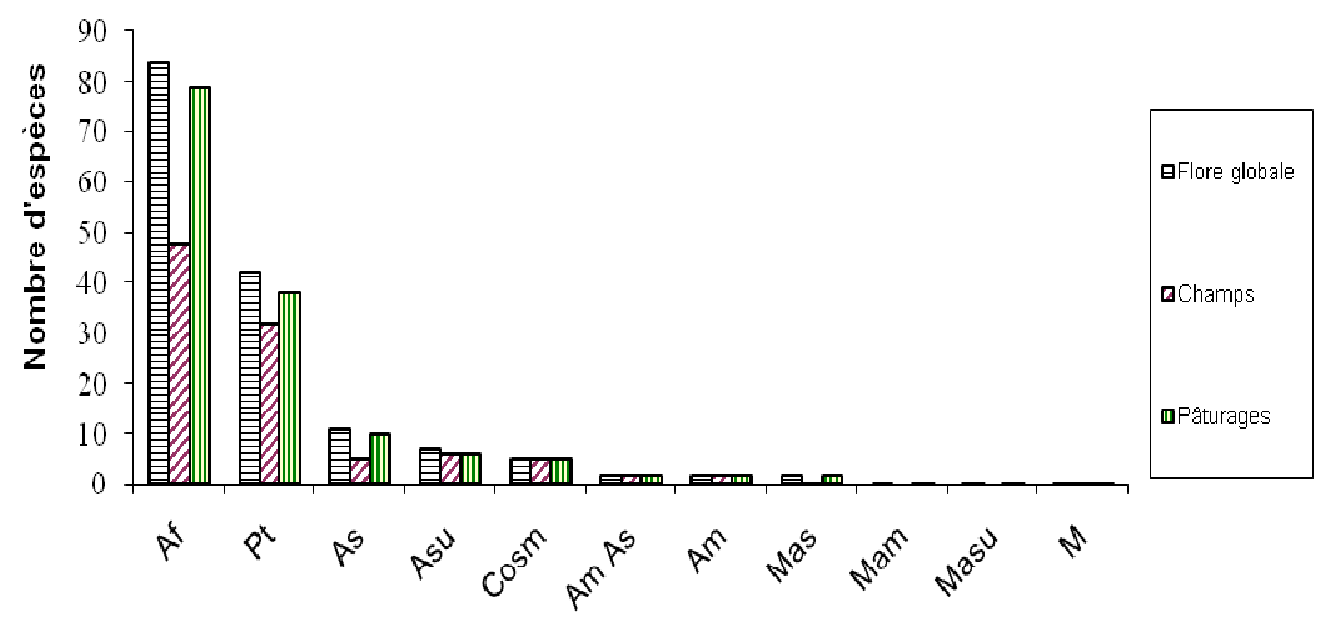

Répartitions géographiques

Figure 4 : Répartition géographique des espèces recensées dans les systèmes agropastoraux. Africaines (Af); Pantropicales (Pt); Asiatiques (As); Australiennes (Asu); Cosmopolites (Cosm); Américaines et asiatiques (AmAs), Américaines (Am); Afro- malgaches et asiatiques (Mas); Afro- malgaches asiatiques et australiennes (Masu) ; Afro-malgaches et américaines (Mam); Afro- malgaches (M). 
Tableau 1: Liste des espèces recensées dans les champs et pâturages avec des indications sur leur type biologique (T.B) et leur répartition géographique (R.G).

\begin{tabular}{|c|c|c|c|c|c|}
\hline Familles & Espèces & Champs & Pâturages & T.B & R.G \\
\hline \multirow[t]{4}{*}{ Acanthaceae } & Asystasia gangetica (L.) T.Anders. & & + & $\mathrm{T}$ & Af \\
\hline & Blepharis maderaspatensis (L.) Heyne & & + & $\mathrm{T}$ & As \\
\hline & Dyschoriste perrottetii (Nees) O. Kze. & & + & $\mathrm{T}$ & Af \\
\hline & Nelsonia canescens (Lam.) Spreng & + & + & $\mathrm{T}$ & Af \\
\hline \multirow[t]{2}{*}{ Amaranthaceae } & Achyranthes aspera $\mathrm{L}$. & & + & $\mathrm{T}$ & Cosm \\
\hline & Pandiaka angustifolia (Vahl.) Hepper & + & + & $\mathrm{T}$ & Af \\
\hline Amaryllidaceae & Haemanthus multiflorus Martyn & & + & G & Af \\
\hline \multirow[t]{2}{*}{ Annonaceae } & Annona glauca Sc.\& Th. & & + & $\mathrm{P}$ & Af \\
\hline & Annona senegalensis Pers. & + & + & $\mathrm{P}$ & Af \\
\hline Araceae & Amorphophallus aphyllus (Hook.) Hutch. & & + & G & Af \\
\hline \multirow[t]{2}{*}{ Asclepiadaceae } & Leptadenia hastata (Pers.) Decne. & + & + & $\mathrm{T}$ & Af \\
\hline & Pergularia daemia (Forssk.) Chiov & + & + & $\mathrm{H}$ & Af \\
\hline \multirow[t]{4}{*}{ Asteraceae } & Acanthospermum hispidum DC. & + & + & $\mathrm{T}$ & $\mathrm{Pt}$ \\
\hline & Blainvillea gayana Cass. & & + & $\mathrm{T}$ & Af \\
\hline & Blumea mollis (D.Don) Merri. & + & & $\mathrm{T}$ & $\mathrm{Pt}$ \\
\hline & Vernonia perrottetii Sch.Bip & + & & $\mathrm{T}$ & $\mathrm{Pt}$ \\
\hline Bombacaceae & Adansonia digitata $\mathrm{L}$. & & + & $\mathrm{P}$ & Af \\
\hline \multirow[t]{5}{*}{ Caesalpiniaceae } & Cassia absus L. & + & + & $\mathrm{T}$ & Af \\
\hline & Cassia mimosoides $\mathrm{L}$. & + & + & $\mathrm{T}$ & Af \\
\hline & Cassia obtusifolia $\mathrm{L}$. & + & + & $\mathrm{T}$ & $\mathrm{Pt}$ \\
\hline & Cassia occidentalis L. & + & + & $\mathrm{T}$ & $\mathrm{Pt}$ \\
\hline & Dialium guineense Willd. & & + & $\mathrm{P}$ & Af \\
\hline Caryophyllaceae & Polycarpaea linearifolia $D C$. & & + & $\mathrm{T}$ & Af \\
\hline Chrysobalanaceae & Neocarya macrophylla (Sabine) Prance & + & + & $\mathrm{P}$ & $\mathrm{Pt}$ \\
\hline \multirow[t]{3}{*}{ Combretaceae } & Combretum micranthum G.Don & & + & $\mathrm{T}$ & Af \\
\hline & Guiera senegalensis J.F.Gmel. & & + & $\mathrm{T}$ & Af \\
\hline & Uvaria chamea P. Beauv. & & + & $\mathrm{T}$ & Af \\
\hline \multirow[t]{2}{*}{ Commelinaceae } & Commelina benghalensis L. & + & + & $\mathrm{T}$ & As \\
\hline & Commelina umbellata Thonn. & + & + & $\mathrm{T}$ & As \\
\hline \multirow[t]{8}{*}{ Convolvulaceae } & Ipomoea asarifolia (Desr.) R. \& Sch. & + & + & $\mathrm{C}$ & $\mathrm{Pt}$ \\
\hline & Ipomoea eriocarpa $\mathrm{R} . \mathrm{Br}$. & & + & $\mathrm{T}$ & Masu \\
\hline & Ipomoea heterotricha F. Didr. & + & + & $\mathrm{T}$ & Asu \\
\hline & Ipomoea pes-tigridis $\mathrm{L}$. & + & + & $\mathrm{T}$ & Asu \\
\hline & Ipomoea vagans Bak. & & + & $\mathrm{T}$ & Af \\
\hline & Merremia aegyptiaca (L.) Urban & + & + & $\mathrm{T}$ & Af \\
\hline & Merremia pinnata (Choisy) Hall. & + & + & $\mathrm{T}$ & Af \\
\hline & Merremia tridentata (L.) Hallier & + & + & $\mathrm{T}$ & Af \\
\hline Cucurbitaceae & Mukia maderaspatana (L.) Roem. & & + & $\mathrm{T}$ & Asu \\
\hline \multirow[t]{7}{*}{ Cyperaceae } & Bulbostylis barbata (Rottb.) Kunth & + & + & $\mathrm{T}$ & $\mathrm{Pt}$ \\
\hline & Bulbostylis hispidula (Vahl) Haines & + & + & $\mathrm{T}$ & Af \\
\hline & Cyperus bulbosus Vahl. & & + & $\mathrm{T}$ & Af \\
\hline & Cyperus cuspidatus Kunth & + & + & $\mathrm{H}$ & $\mathrm{Pt}$ \\
\hline & Cyperus cyperoides (L.) Kuntze. & & + & $\mathrm{T}$ & $\mathrm{Pt}$ \\
\hline & Cyperus esculentus $\mathrm{L}$. & + & + & G & Cosm \\
\hline & Cyperus margaritaceus Vahl. & + & + & $\mathrm{T}$ & Af \\
\hline
\end{tabular}




\begin{tabular}{|c|c|c|c|c|c|}
\hline & Cyperus rotundus $\mathrm{L}$. & + & + & G & Cocm \\
\hline & Cyperus sphacelatus Rottb. & + & + & $\mathrm{T}$ & $\mathrm{Pt}$ \\
\hline & Kyllinga odorata Vahl. & + & + & G & Af \\
\hline & Mariscus ligularis (L.) Urb. & & + & $\mathrm{T}$ & $\mathrm{Pt}$ \\
\hline \multirow[t]{6}{*}{ Euphorbiaceae } & Chrozophora senegalensis (Lam.) A. Juss. & + & + & $\mathrm{T}$ & Af \\
\hline & Euphorbia aegyptiaca Boiss. & + & & $\mathrm{T}$ & Af \\
\hline & Euphorbia hirta L. & + & + & $\mathrm{T}$ & $\mathrm{Pt}$ \\
\hline & Micrococca mercurialis (L.) Benth. & & + & $\mathrm{T}$ & Mas \\
\hline & Phyllanthus niruri auct. Afric. & & + & $\mathrm{T}$ & Af \\
\hline & Phyllanthus pentandrus Sch. \& Th & & + & $\mathrm{T}$ & Af \\
\hline \multirow[t]{19}{*}{ Fabaceae } & Alysicarpus ovalifolius (Schum.) Léonaed & + & + & $\mathrm{T}$ & $\mathrm{Pt}$ \\
\hline & Crotalaria goreensis Guill. et Perr. & + & + & $\mathrm{T}$ & Af \\
\hline & Crotalaria retusa $\mathrm{L}$. & + & + & $\mathrm{T}$ & $\mathrm{Pt}$ \\
\hline & Desmodium hirtum G.\& Perr. & + & + & $\mathrm{T}$ & Af \\
\hline & Desmodium ospriostreblum Chiov. & & + & $\mathrm{T}$ & Af \\
\hline & Indigofera astragalina DC. & + & + & $\mathrm{T}$ & Af \\
\hline & Indigofera berhautiana Gillett & & + & $\mathrm{T}$ & Af \\
\hline & Indigofera dendroides Jacq. & + & + & $\mathrm{T}$ & Af \\
\hline & Indigofera nummulariifolia (L.) Liv. & + & + & $\mathrm{T}$ & Af \\
\hline & Indigofera pilosa Poir. & + & + & $\mathrm{T}$ & Af \\
\hline & Sesbania pachycarpa DC. & + & + & $\mathrm{P}$ & Asu \\
\hline & Stylosanthes erecta P.Beauv & + & + & $\mathrm{T}$ & Af \\
\hline & Stylosanthes fruticosa (Retz.) Alton & + & + & $\mathrm{T}$ & Af \\
\hline & Tephrosia latyroides G.\& Perr. & + & + & $\mathrm{T}$ & Af \\
\hline & Tephrosia linearis (Willd.) Pers. & + & + & $\mathrm{T}$ & Af \\
\hline & Tephrosia lupinifolia DC. & + & + & $\mathrm{T}$ & Af \\
\hline & Tephrosia pedicellata Bak. & & + & $\mathrm{T}$ & $A f$ \\
\hline & Vigna unguiculata (L.) Walp. & + & & $\mathrm{T}$ & $\mathrm{Pt}$ \\
\hline & Zornia glochidiata Reichb. & + & + & $\mathrm{T}$ & $\mathrm{Pt}$ \\
\hline Icacinaceae & Icacina senegalensis A. Juss & & + & G & Af \\
\hline Lamiaceae & Hyptis suaveolens (L.) Poit. & + & + & $\mathrm{T}$ & Am As \\
\hline Liliaceae & Scilla sudanica A.Chev. & + & + & G & Af \\
\hline \multirow[t]{11}{*}{ Malvaceae } & Hibiscus asper Hook. f. & + & + & $\mathrm{T}$ & Af \\
\hline & Hibiscus diversifolius Jacq. & + & & $\mathrm{T}$ & Af \\
\hline & Hibiscus mechowii Garcke. & + & & $\mathrm{T}$ & Af \\
\hline & Hibiscus sabdarifa L. & + & + & $\mathrm{T}$ & Af \\
\hline & Hibiscus surrattensis L. & & + & $\mathrm{T}$ & Af \\
\hline & Sida cordifolia $\mathrm{L}$. & + & + & $\mathrm{T}$ & Af \\
\hline & Sida linifolia Juss. & + & + & $\mathrm{T}$ & Af \\
\hline & Sida rhombifolia $\mathrm{L}$. & + & + & $\mathrm{T}$ & $\mathrm{Pt}$ \\
\hline & Sida stipulata Cav. & + & + & $\mathrm{T}$ & $\mathrm{Pt}$ \\
\hline & Sida urens L. & & + & $\mathrm{T}$ & $\mathrm{Pt}$ \\
\hline & Urena lobata $\mathrm{L}$ & + & + & $\mathrm{T}$ & Af \\
\hline Meliaceae & Azadirachta indica A. Juss. & & + & $\mathrm{P}$ & As \\
\hline Menispermaceae & Cissampelos mucronata A. Rich. & & + & $\mathrm{P}$ & Af \\
\hline \multirow[t]{4}{*}{ Mimosaceae } & Parkia biglobosa (Jacq.) R.Br.ex G.Don f. & + & + & $\mathrm{P}$ & Af \\
\hline & Acacia sieberiana DC. & & + & $\mathrm{P}$ & Af \\
\hline & Faidherbia albida (Del.) A. Chev. & + & + & $\mathrm{P}$ & Af \\
\hline & Dichrostachys cinerea (L.) Wight \& Arn. & + & + & $\mathrm{P}$ & Af \\
\hline Moraceae & Ficus vogelii Miq. & & + & $\mathrm{P}$ & Af \\
\hline
\end{tabular}




\begin{tabular}{|c|c|c|c|c|c|}
\hline Nyctaginaceae & Boerhaavia erecta $\mathrm{L}$. & & + & $\mathrm{T}$ & $\mathrm{Pt}$ \\
\hline Onagraceae & Ludwigia abyssinica A. Rich. & + & & $\mathrm{H}$ & $\mathrm{Pt}$ \\
\hline Passifloraceae & Passiflora foetida $\mathrm{L}$. & + & + & $\mathrm{T}$ & Am \\
\hline \multirow[t]{42}{*}{ Poaceae } & Acroceras amplectens Stapf. & + & & $\mathrm{T}$ & Af \\
\hline & Andropogon gayanus Kunth. & + & + & $\mathrm{H}$ & Af \\
\hline & Aristida adscensionis $L$. & & + & $\mathrm{T}$ & $\mathrm{Pt}$ \\
\hline & Aristida sieberiana Trin. & & + & $\mathrm{T}$ & $\mathrm{Pt}$ \\
\hline & Brachiaria comata (A. Rich.) Stapf & & + & $\mathrm{T}$ & Af \\
\hline & Brachiaria distichophylla (Trin.)Stapf. & & + & $\mathrm{T}$ & Af \\
\hline & Brachiaria jubata (Fig. \& De Notaris) Stapf & & + & $\mathrm{H}$ & Af \\
\hline & Brachiaria lata (Schum.) Hubb. & + & + & $\mathrm{T}$ & $\mathrm{Pt}$ \\
\hline & Brachiaria stigmatisata (Mez) Stapf & + & + & $\mathrm{T}$ & Af \\
\hline & Brachiaria villosa (Lam.) A.Camus & + & + & $\mathrm{T}$ & $\mathrm{Pt}$ \\
\hline & Brachiaria xantholeuca (Sch.) Stapf & & + & $\mathrm{T}$ & $\mathrm{Pt}$ \\
\hline & Cenchrus biflorus Roxb. & & + & $\mathrm{T}$ & As \\
\hline & Chloris barbata $\mathrm{Sw}$. & + & + & $\mathrm{T}$ & As \\
\hline & Chloris prieurii Kunth & & + & $\mathrm{T}$ & As \\
\hline & Chloris pilosa Schum. & + & + & $\mathrm{T}$ & Af \\
\hline & Cynodon dactylon (L.) Pers. & + & + & $\mathrm{H}$ & Cosm \\
\hline & Dactyloctenium aegyptium (L.) Willd. & + & + & $\mathrm{T}$ & $\mathrm{Pt}$ \\
\hline & Digitaria ciliaris (Retz.) Koeler & + & + & $\mathrm{T}$ & $\mathrm{Pt}$ \\
\hline & Digitaria horizontalis Willd. & + & + & $\mathrm{T}$ & $\mathrm{Pt}$ \\
\hline & Echinochloa colona (L.) Link & & + & $\mathrm{T}$ & $\mathrm{Pt}$ \\
\hline & Eleusine indica (L.) Gaertn. & + & + & $\mathrm{T}$ & $\mathrm{Pt}$ \\
\hline & Eragrostis ciliaris var. ciliaris (L.) R. Br. & + & + & $\mathrm{T}$ & $\mathrm{Pt}$ \\
\hline & Eragrostis gangetica (Roxb) Steud. & + & + & $\mathrm{T}$ & As \\
\hline & Eragrostis japonica (Thunb.) Trinius. & & + & $\mathrm{T}$ & As \\
\hline & Eragrostis pilosa (L.) P. Beauv. & + & + & $\mathrm{T}$ & Cosm \\
\hline & Eragrostis squamata (Lam.) Steud. & + & + & $\mathrm{H}$ & Af \\
\hline & Eragrostis tenella (L.) Roem.\& Sch. & & + & $\mathrm{T}$ & Af \\
\hline & Eragrostis tremula Steud. & & + & $\mathrm{T}$ & As \\
\hline & Loudetia annua (Stapf) Hubb. & + & + & $\mathrm{T}$ & Af \\
\hline & Panicum anabaptistum Steud. & & + & $\mathrm{C}$ & Af \\
\hline & Panicum laetum kunth & & + & $\mathrm{T}$ & Af \\
\hline & Panicum parvifolium Lam. & & + & $\mathrm{H}$ & M Am \\
\hline & Panicum subalbidum Kunth & & + & $\mathrm{H}$ & Af \\
\hline & Panicum walense $\mathrm{Mez}$ & & + & $\mathrm{T}$ & As \\
\hline & Paspalum orbuculare G. Forst. & + & & $\mathrm{T}$ & $\mathrm{Pt}$ \\
\hline & Paspalum scrobiculatum L. & + & & $\mathrm{H}$ & Mas \\
\hline & Pennisetum violaceum (Lam.) L. Rich. & + & + & $\mathrm{T}$ & Asu \\
\hline & Perotis patens Grand. & + & & $\mathrm{T}$ & $\mathrm{M}$ \\
\hline & Perotis scabra Will.ex Trin. & + & + & $\mathrm{T}$ & $\mathrm{Pt}$ \\
\hline & Setaria barbata Kunth. & & + & $\mathrm{T}$ & $\mathrm{Pt}$ \\
\hline & Setaria pumila (Poir.) Roem. \& Schult. & + & + & $\mathrm{T}$ & Asu \\
\hline & Sporobolus pyramidalis P. Beauv. & & + & $\mathrm{H}$ & Am \\
\hline \multirow[t]{4}{*}{ Rubiaceae } & Kohautia senegalensis Cham. \& Sch. & + & + & $\mathrm{T}$ & Af \\
\hline & Mitracarpus villosus (Sw.) DC. & + & + & $\mathrm{T}$ & Am As \\
\hline & Oldenlandia corymbosa $\mathrm{L}$. & + & + & $\mathrm{T}$ & $\mathrm{Pt}$ \\
\hline & Oldenlandia herbacea (L.) Roxb. & + & + & $\mathrm{T}$ & $\mathrm{Pt}$ \\
\hline
\end{tabular}




\begin{tabular}{|c|c|c|c|c|c|}
\hline & Spermacoce ruelliae DC. & + & + & $\mathrm{T}$ & Af \\
\hline & Spermacoce stachydea DC. & + & + & $\mathrm{T}$ & Af \\
\hline & Spermacoce verticillata $\mathrm{L}$. & + & + & $\mathrm{T}$ & Af \\
\hline & Spermacoce radiata (DC.) & + & + & $\mathrm{T}$ & Af \\
\hline Sapindaceae & Allophyllus cobbe (L.) Raeuch. & & + & $\mathrm{P}$ & Af \\
\hline Scrofulariaceae & Scoparia dulcis $\mathrm{L}$ & + & + & $\mathrm{T}$ & $\mathrm{Pt}$ \\
\hline \multirow[t]{3}{*}{ Sterculiaceae } & Melochia corchorifolia $\mathrm{L}$. & + & + & $\mathrm{T}$ & Af \\
\hline & Melochia melissifolia Benth. & + & + & $\mathrm{T}$ & Af \\
\hline & Waltheria indica $\mathrm{L}$. & + & + & $\mathrm{T}$ & $\mathrm{Pt}$ \\
\hline \multirow[t]{3}{*}{ Tiliaceae } & Corchorus aestuans L. & & + & $\mathrm{T}$ & $\mathrm{Pt}$ \\
\hline & Corchorus tridens L. & + & + & $\mathrm{T}$ & Asu \\
\hline & Triumfetta pentendra A. Rich. & & + & $\mathrm{T}$ & $\mathrm{Pt}$ \\
\hline Verbenacea & Clerodendrum capitatum (Willd.) Sc.\& Th. & & + & $\mathrm{P}$ & Af \\
\hline Vitaceae & Ampelocissus pentaphylla (Guill.\& Perr.). & & + & $\mathrm{H}$ & Af \\
\hline
\end{tabular}

Tableau 2 : Répartition par famille des espèces de la flore des systèmes agropastoraux.

\begin{tabular}{|c|c|c|c|c|c|c|}
\hline \multirow[t]{3}{*}{ Familles } & \multicolumn{2}{|c|}{ Flore globale } & \multicolumn{2}{|c|}{ Flore des Champs } & \multicolumn{2}{|c|}{ Flore des pâturages } \\
\hline & N.E & $\%$ & N.E & $\%$ & N.E & $\%$ \\
\hline & & Famille & & Famille & & Famille \\
\hline Poaceae (M) & 42 & 26,6 & 23 & 22,55 & 38 & 25,85 \\
\hline Fabaceae (D) & 19 & 12,02 & 16 & 15,7 & 18 & 12,25 \\
\hline Cyperaceae (M) & 11 & 6,96 & 9 & 8,82 & 11 & 7,5 \\
\hline Malvaceae (D) & 11 & 6,96 & 9 & 8,82 & 9 & 6,12 \\
\hline Convolvulaceae (D) & 8 & 5,06 & 6 & 5,88 & 8 & 5,44 \\
\hline Rubiaceae (D) & 8 & 5,06 & 8 & 7,84 & 8 & 5,44 \\
\hline Euphorbiaceae (D) & 6 & 3,8 & 3 & 2,94 & 5 & 3,4 \\
\hline Caesalpiniaceae (D) & 5 & 3,2 & 4 & 3,93 & 5 & 3,4 \\
\hline Acanthaceae (D) & 4 & 2,53 & 1 & 0,98 & 4 & 2,72 \\
\hline Asteraceae (D) & 4 & 2,53 & 3 & 2,94 & 2 & 1,36 \\
\hline Mimosaceae (D) & 4 & 2,53 & 3 & 2,94 & 4 & 2,72 \\
\hline Combretaceae (D) & 3 & 1,9 & 1 & 0,98 & 3 & 2,04 \\
\hline Sterculiaceae (D) & 3 & 1,9 & 3 & 2,94 & 3 & 2,04 \\
\hline Tiliaceae (D) & 3 & 1,9 & 1 & 0,98 & 3 & 2,04 \\
\hline Amaranthaceae (D) & 2 & 1,27 & 1 & 0,98 & 2 & 1,36 \\
\hline Annonaceae (D) & 2 & 1,27 & 1 & 0,98 & 2 & 1,36 \\
\hline Asclepiadaceae (D) & 2 & 1,27 & 2 & 1,96 & 2 & 1,36 \\
\hline Commelinaceae (M) & 2 & 1,27 & 2 & 1,96 & 2 & 1,36 \\
\hline Amaryllidaceae (M) & 1 & 0,63 & 0 & 0 & 1 & 0,68 \\
\hline Araceae $(\mathrm{M})$ & 1 & 0,63 & 0 & 0 & 1 & 0,68 \\
\hline Bombacaceae (D) & 1 & 0,63 & 0 & 0 & 1 & 0,68 \\
\hline Caryophyllaceae (D) & 1 & 0,63 & 0 & 0 & 1 & 0,68 \\
\hline Chrysobalanaceae (D) & 1 & 0,63 & 1 & 0,98 & 1 & 0,68 \\
\hline Cucurbitaceae (D) & 1 & 0,63 & 0 & 0 & 1 & 0,68 \\
\hline
\end{tabular}




\begin{tabular}{lcccccc}
\hline Icacinaceae (D) & 1 & 0,63 & 0 & 0 & 1 & 0,68 \\
Lamiaceae (D) & 1 & 0,63 & 1 & 0,98 & 1 & 0,68 \\
Liliaceae (M) & 1 & 0,63 & 1 & 0,98 & 1 & 0,68 \\
Meliaceae (D) & 1 & 0,63 & 0 & 0 & 1 & 0,68 \\
Menispermaceae (D) & 1 & 0,63 & 0 & 0 & 1 & 0,68 \\
Moraceae (D) & 1 & 0,63 & 0 & 0 & 1 & 0,68 \\
Nyctaginaceae (D) & 1 & 0,63 & 0 & 0 & 1 & 0,68 \\
Onagraceae (D) & 1 & 0,63 & 1 & 0,98 & 0 & 0 \\
Passifloraceae (D) & 1 & 0,63 & 1 & 0,98 & 1 & 0,68 \\
Sapindaceae (D) & 1 & 0,63 & 0 & 0 & 1 & 0,68 \\
Scrofulariaceae (D) & 1 & 0,63 & 1 & 0,98 & 1 & 0,68 \\
Verbenaceae (D) & 1 & 0,63 & 0 & 0 & 1 & 0,68 \\
Vitaceae (D) & 1 & 0,63 & 0 & 0 & 1 & 0,68 \\
Total & 158 & 100 & 102 & 100 & 147 & 100 \\
\hline \multicolumn{1}{c}{ D : Dicotylédones ; M : Monocotylédones ; N. E : Nombre d'Espèces ; \% Famille : pourcentage. }
\end{tabular}

Tableau 3 : Types biologiques des espèces dans les champs et des pâturages.

\begin{tabular}{lcccc}
\hline & \multicolumn{2}{c}{ Champs } & \multicolumn{2}{c}{ Pâturages } \\
\cline { 2 - 5 } Types biologiques & N.E & \% & N.E & \% \\
\hline T & 84 & 82,35 & 113 & 76,87 \\
P & 6 & 5,88 & 15 & 10,20 \\
H & 7 & 6,86 & 10 & 6,80 \\
G & 4 & 3,92 & 7 & 4,76 \\
C & 1 & 0,98 & 2 & 1,36 \\
Total & 102 & 100,00 & 147 & 100,00 \\
\hline
\end{tabular}

Tableau 4 : Répartition géographique des espèces recensées dans les champs et les pâturages.

\begin{tabular}{lcccc}
\hline & \multicolumn{2}{c}{ Champs } & \multicolumn{2}{c}{ Pâturages } \\
\hline & N.E & \% & N.E & \% \\
Af & 52 & 50,98 & 82 & 55,78 \\
Pt & 31 & 30,39 & 37 & 25,17 \\
As & 4 & 3,92 & 10 & 6,80 \\
Asu & 6 & 5,88 & 7 & 4,76 \\
Cosm & 4 & 3,92 & 5 & 3,40 \\
AmAs & 2 & 1,96 & 2 & 1,36 \\
Mas & 1 & 0,98 & 1 & 0,68 \\
Masu & 0 & 0,00 & 1 & 0,68 \\
Am & 1 & 0,98 & 1 & 0,68 \\
Mam & 0 & 0,00 & 1 & 0,68 \\
M & 1 & 0,98 & 0 & 0,00 \\
Total & 102 & 100,00 & 147 & 100,00 \\
\hline
\end{tabular}




\section{DISCUSSION}

\section{Spectre taxonomique}

Dans la flore globale des systèmes agropastoraux, en considérant les familles ayant 8 espèces au moins comme les plus importantes, 6 Familles regroupent plus des $3 / 5$, soit $62,66 \%$ des espèces (Tableau 2). Ce sont par ordre décroissante les Poaceae, les Fabaceae, les Cyperaceae, les Malvaceae, les Convolvulaceae et les Rubiaceae. A titre de comparaison, la prédominance des 6 familles de la flore de Mlomp (Tableau 2) est relativement identique à celle de la flore du Bassin arachidier (Noba et al., 2004) et des Niayes (Sarr et al., 2007) respectivement, Poaceae $(26,6 \%$ contre $20 \%$ et $23 \%$ ), les Fabaceae $(12,02 \%$ contre $8,8 \%$ et $11,5 \%)$, les Cyperaceae $(6,96 \%$ contre $6,4 \%$ et $6 \%$ ), les Malvaceae $(6,96 \%$ contre $4,8 \%$ et $2,3 \%)$, les Convolvulaceae $(5,06 \%$ contre $7,2 \%$ et $6 \%)$ et Rubiaceae (5,06\% contre $6,4 \%$ et $3,1 \%) .12$ familles dont le nombre d'espèces est compris entre 2 et 6 représentent $25,34 \%$ et 19 familles représentées par une seule espèce, totalisent $12 \%$ des espèces de la flore globale.

Comparée à la flore globale du Sénégal (Bâ et al., 2001), la flore adventice des cultures vivrières dans le sud du Bassin arachidier (Sénégal) (Noba et al., 2004), la flore adventice des cultures d'oignon dans la zone péri-urbaine de Dakar (Niayes) Sénégal (Sarr et al., 2007), la flore des systèmes agropastoraux de Mlomp (Tableau 2), présente des proportions comparables entre le Monocotylédones et les Dicotylédones. Les Dicotylédones représentent $63,29 \%$ contre $69,5 \%, 68,4 \%$ et $66,4 \%$ respectivement pour la flore globale du Sénégal, du Bassin arachidier et des Niayes. Les Monocotylédones représentent 36,71\% contre $28,8 \%, 31,2 \%$ et $32,1 \%$ respectivement la flore globale du Bassin arachidier et des Niayes. Les proportions des Dicotylédones et des monocotylédones de Mlomp sont plus proches de celles des Niayes. Cela pourrait s'expliquer par le fait que la Basse Casamance et les Niayes présentent les caractéristiques d'une zone humide favorable au développement d'une flore diversifiée. Ces proportions sont différentes de celles observées dans la flore du Burkina Faso constituée de $71 \%$ pour les Dicotylédones et $29 \%$ pour les Monocotylédones (Guinko, 1984). Ces différences sont dues probablement au fait que la Basse Casamance appartient à un type de climat soudanoguinéen (Pelissier, 1983) alors que le climat du Burkina Faso est du type soudano- sahélien (Guinko et al., 1998).

On peut par ailleurs noter dans certaines familles des espèces ligneuses (Combretaceae, Chrysobalanaceae et des Mimosaceae) arbustives. La présence de ces familles suggèrerait une savanisation progressive de la végétation due aux effets conjugués des facteurs climatiques et anthropiques (Noba et al., 2004).

Le cas de Guira senegalensis est plus significatif car selon (Vanden Verghen, 1998), sa présence est son extension seraient liées à l'introduction et à la vulgarisation de la culture de l'arachide. Et pour le cas de Faidherbia albida, Dichrostachys cinerea et Néocarya macrophylla, leur extension pourraient être liées à la dissémination des graines, par les déjections de certains animaux qui pourraient contenir des graines issues des fruits consommés.

Les Légumineuses (Caesalpiniaceae, Fabaceae et Mimosaceae) regroupent 18,05\% des familles de la flore. Cette proportion est comparable à celle de la flore du Bassin arachidier $(14,4 \%)$ et celle des Niayes $(14,5 \%)$. Ces résultats sont en relation avec ceux de Grouzis et al. (1998) qui montrent que les proportions des Légumineuses dans la strate herbacée des systèmes écologiques diminuent du sud au nord du sénégal.

Dans cette flore de Mlomp, est signalée la seule espèce Icacina senegalensis de la famille des Icacinaceae signalée dans la flore du Sénégal.

\section{Spectre biologique}

La dominance de ces espèces annuelles pourrait s'expliquer par leur dissémination dans le milieu par le biais des facteurs climatiques tels que le vent, l'eau ou par les animaux. En effet, les thérophytes adaptent 
leur cycle de développement à la période favorable qui correspond à la saison des pluies. Elles ont un cycle très court, parfois de quelques semaines comme chez les espèces du genre Boerhavia (Noba et al., 1994) et du genre Corchorus (Mbaye et al., 2001). D'autres traits concernant l'annualité des plantes, la croissance, la résistance aux perturbations ou encore la longévité peuvent caractériser les communautés d'adventices ou de plantes localisées dans des environnements changeants. Ainsi, les plantes annuelles ayant une croissance rapide montrent davantage de caractéristiques nécessaires pour se rétablir rapidement que les plantes vivaces, à croissance lente, ligneuses ou rhizomateuses (Hawes et al., 2010). Ces thérophytes sont relativement moins représentés dans la flore de Mlomp $(77,21 \%)$ que dans la flore du Bassin arachidier 85,6\% (Noba et al., 2004) et des Niayes 87\% (Sarr et al., 2007). Cette différence est probablement due à la répartition spatiale de la pluviométrie dans le pays qui diminue du sud vers le nord.

Les autres types biologiques sont les phanérophytes $(9,49 \%)$, les hémicrytophytes $(7,6 \%)$, les géophytes $(4,43 \%)$ et les chaméphytes $(1,27 \%)$. Les phanérophytes sont constituées essentiellement des espèces ligneuses (Combretaceae, de Légumineuses arbustives, de Chrysobalanaceae et de Meliaceae). L'adaptation en milieu sec des hémicrytophytes, des géophytes et des chaméphytes expliqueraient leurs faibles proportions (Garba, 1984).

\section{Spectre chorologique}

Sur le plan de la répartition géographique des espèces, la dominance des espèces africaines est probablement due à des systèmes de résistance aux perturbations dans cette localité. Cette dominance pourra s'expliquer par le fait que les espèces africaines soient plus adaptées aux conditions bioclimatiques du milieu que les autres espèces étrangères (Noba et al., 2004). Les espèces afro-asiatiques $(6,96 \%)$; australiennes $(4,43 \%)$; cosmopolites $(3,16 \%)$; américaines $(1,26 \%)$, américaines et asiatiques, $(1,26 \%)$, afro-malgaches et asiatiques $(1,26 \%)$; pour les espèces afro-malgaches et américaines $(0,63 \%)$ afro-malgaches asiatiques et australiennes $(0,63 \%)$ et enfin afro-malgaches $(0,63 \%)$ sont également représentées dans cette flore.

Influence du système d'exploitation des terres sur la structure de la flore

La différence spécifique entre les champs et pâturage s'expliquerait par les activités menées dans ces différentes zones. En effet, dans les champs, le labour avec un instrument traditionnel appelé «Kadiandou» en diola, qui consiste à retourner le sol, enfouit les graines des plantes en des profondeurs de plus de $10 \mathrm{~cm}$. Ceci pourrait empêcher d'une part, la germination ou levée des adventices (Bassène, 2008). D'autre part, ce labour pourrait entraîner la destruction des organes souterrains de certaines espèces par des coupes et leur exposition en air sous le soleil. Le désherbage successif contribue aussi à une réduction de la densité et de la diversité des adventices dans les champs pour éviter la concurrence nutritionnelle entre ces adventices et les plantes cultivées (Noba, 2002 ; Mbaye, 2013).

En revanche, au niveau des pâturages, le sol n'étant pas labouré ni désherbé, les jeunes plants issus des germinations des semences peuvent bien se développer et donner une flore diversifiée. Certaines espèces sont retrouvées uniquement dans les champs. Cela expliquerait le fait que ces espèces sont des espèces de milieu humide, car les champs sont parfois inondés en plaine saison des pluies tandis que les zones de pâturages ne le sont pas. Par exemple, on peut citer une espèce de la famille des Onagraceae (Ludwigia abissinica) qui a été retrouveé uniquement dans les champs, plus précisément dans les plaques d'eau.

Influence du système d'exploitation sur les types biologiques des espèces

Les espèces thérophytes sont dominantes aussi bien dans les champs que dans les pâturages. Dans les pâturages, ces thérophytes sont suivies des phanérophytes, des hémicrytophytes, des géophytes et des chaméphytes. Par contre, dans les champs, on 
note les hémicrytophytes avant les phanérophytes, puis les géophytes et les chaméphytes (Figure 3). Cette différence de position entre les phanérophytes et les hémicrytophytes dans les champs et les pâturages pourrait s'expliquer par les différentes activités menées dans ces zones. Dans les champs, les phanérophytes sont coupés volontairement par les paysans (Noba et al., 2004). Ces coupes permettent de dégager les champs pour éviter les ombrages des cultures par ces arbres et qui empêchent l'arrivée de la lumière aux plantes cultivées. Ainsi, les quelques phanérophytes qui y existent sont constitués essentiellement des espèces ligneuses originelles qui n'ont pu être arrachées par les paysans au cours des défrichements et des labours à cause de leur profond enracinement (Noba et al., 2004). L'intensification de l'agriculture favorise l'abondance et la richesse de ces espèces végétales annuelles (Liira et al., 2008). Ces plantes possèdent des traits nécessaires à leur survie dans ces zones cultivées comme une régénération annuelle de la banque de graines, une période courte de floraison et un renouvellement rapide des populations. Ces résultats rappellent celles de Hawes et al. (2010). Selon Lindborg (2007), ces espèces vivant peu de temps sont plus vulnérables mais répondent plus rapidement à l'adaptation aux conditions du milieu perturbé que les espèces vivant plus longtemps.

\section{Influence du système d'exploitation sur la répartition géographique des espèces}

Sur le plan de la répartition géographique dans le monde des espèces répertoriées, l'ordre d'importance des espèces du spectre chorologique des champs est similaire à celui des pâturages, excepté les espèces afro-malgaches et américaines et afromalgaches asiatiques et australiennes qui ne sont pas signalées dans zone. L'absence de ces espèces dans les champs, suggérerait soit par l'absence d'introduction ou bien ces espèces ont été détruites au moment du labour ou les périodes de désherbage (Bassène, 2014).

\section{Conclusion}

Ce travail a donc permis de caractériser qualitativement la flore des systèmes agropastoraux de Mlomp. Il montre que la flore des champs présente approximativement la même structure que la flore des pâturages, à quelques différences près.

Il ressort de cette étude que cette flore est fortement diversifiée avec au moins 158 espèces, réparties en 91 genres réunis en 37 familles. Les familles les plus représentées sont les Poaceae (26,6\%), les Fabaceae $(12,02 \%)$, les Cyperaceae $(6,96 \%)$, les Malvaceae $(6,96 \%)$ et les Convolvulaceae $(5,06 \%)$ et les Rubiaceae $(5,06 \%)$. Douze (12) autres familles sont relativement bien représentées $(10,8 \%)$ avec 6 espèces au maximum. Enfin, 19 autres familles totalisent près de $12 \%$ des espèces et sont représentées chacune par une seule espèce.

Cette flore est constituée de 77,21\% de thérophytes, 9,49\% de phanérophytes, 7,6\% de hémicrytophytes, $4,43 \%$ de géophytes et $1,27 \%$ de chaméphytes.

Elle présente une grande diversité biogéographique, composée pour l'essentiel, d'espèces africaines $(53,2 \%)$, d'espèces pantropicales $(26,58 \%)$ et d'espèces afroasiatiques $(6,96 \%)$. Le reste est constitué d'espèces australiennes $(4,43 \%)$, cosmopolites $(3,16 \%)$, américaines et asiatiques $(1,26 \%)$, américaines (1,26\%), afro-malgaches et asiatiques (1,26\%), afro-malgaches et américaines $\quad(0,63 \%)$, afro-malgaches asiatiques et australiennes $(0,63 \%)$ et afromalgaches $(0,63 \%)$.

La préparation des sols avant la culture et le labour des sols jouent un rôle important dans la distribution de la flore des champs.

\section{REFERENCES}

Bâ AT, Noba K. 2001. Science et changements planétaires. Sécheresse. 12(3) : 149-155.

Bassène C. 2014. La flore adventice dans les cultures de maïs (Zea mays L.) dans le sud du Bassin Arachidier : structure, nuisibilité et mise au point d'un itinéraire de désherbage. Thèse Unique. 
Université Ckeikh Anta Diop de Dakar, Sénégal, p. 165.

Bassène C. 2008. Hyptis suaveolens L. Poit. (Lamiaceae) dans les systèmes agropastoraux de la communauté rurale de Mlomp: Etude de quelques aspects de la biologie, de l'écologie et proposition de méthodes de contrôle. Mémoire de DEA. Université Cheikh Anta Diop de Dakar. Sénégal, p.108.

Garba M. 1984. Contribution à l'étude de la flore et de la végétation des milieux aquatiques et des sols hydromorphes de l'ouest de la république de Niger de la longitude de dogondoutchi au fleuve Niger. Thèse de Doctorat $3^{\mathrm{e}}$ cycle, Univ. de Bordeaux III, p. 149.

Grousiz M, Diédhiou I. 1998. Importance des légumineuses dans les systèmes écologiques semi-arides du Sénégal. AAU Reports, 39 : 97-111.

Guinko S. 1984. Végétation de la Haute Volta. Thèse de doctorat d'état, Université de Bordeaux III, p. 394.

Guinko S, Ouedraogo M. 1998. La flore du Burkina Faso. In: Atelier sur flore, végétation et biodiversité au Sahel, Bâ AT, Madsen JE, Sambou B (eds). AAU Reports, 39, pp43-65.

Hawes C, Squire GR, Hallett PD, Watson CA, Young M. 2010. Arable plant communities as indicators of farming practice. Agr. Eco. Environment, 138 : 17-26

Le Bourgeois T, Merlier H. 1995. Adventrop : les adventices d'Afrique soudanosahélienne. CIRAD-CA Montpellier.

Lebrun J. 1966. Les formes biologiques dans les végétations tropicales. Bull. Sco. Bot. France. 164- 175.

Lebrun JP, Stork A.1991. Enumération des Plantes à Fleurs d'Afrique Tropicale (vol I, II, III, IV). Edition des conservatoires et jardin botaniques de la ville de Genève : Genève.

Liira J, Schmidt T, Aavik T, Arens P, Augenstein I, Bailey D, Billeter R, Bukacek R, Burel F, De Blust G, De Cock R, Dirksen J, Edwards PJ,
Hamersky R, Herzog F, Klotz S, Kuhn I, Le Coeur D, Miklova P, Roubalova M, Schweiger O, Smulders MJM, Van Wingerden WKRE, Bugter R, Zobel M. 2008. Plant functional group composition and large-scale species richness in European agricultural landscapes. Journal of Vegetation Science, 19: 3 - 14.

Lindborg R. 2007. Evaluating the distribution of plant life history traits relation to current and historical landscape configurations. Ecological Society, Journal of Ecology. Handling Editor: Bryan Foster, 95(3): 555-564.

Mbaye MS. Noba K, Sarr RS, Kane A, Sambou JM, Bâ AT. 2001. Caractères spécifiques d'identification au stade jeune plant d'adventices Sénégalaises du genre Corchorus L. (Tiliaceae). Ann. Bot. Afr. O. 00 (1): 35-42.

Mbaye MS. 2013. Association mil [Pennisetum glaucum (L.) R.Br] et niébé [Vigna unguiculata (L.) Walp.] : arrangement spatiotemporel des cultures, structures, dynamique et concurrence de la flore adventice et proposition d'un itinéraire technique. Thèse de Doctorat d'état. Université Ckeikh Anta Diop de Dakar. p.236.

Merlier H, Montegu T. 1982. Adventices tropicales.

ORSTOM-GERDATENSH.

Noba K, Samb PI, Bâ AT. 1994. Sur quelques caractères macro et micromorphologiques du jeune plant dans la systématique de trois espèces du genre Boerhavia L. (Nyctaginaceae). Bull. Inst. Fond. Afr. Noire C. A. Diop, Dakar, Sér. A, 47: 51-62.

Noba K, Bâ AT, Caussanel J-P, Mbaye MS, Barralis G. 2004. Flore adventice des cultures vivrières dans le sud du Bassin arachidier (Sénégal). Webbia, 59(2): 293-308.

Pelissier P. 1983. Atlas du Sénégal. Jeune Afrique : Paris.

Poilecot P. 1999. Les Poaceae du Niger. Edition des conservatoires et jardin 
botaniques de la ville de Genève : Boissiera.

Raunkier C. 1934. The Life Forms of Plants and Statistical Plants Geography. Clarendron Press: Oxford.

Sarr RS, Mbaye MS, Bâ AT. 2007. La flore adventice des cultures d'oignon dans la zone péri-urbaine de Dakar (Niayes) Sénégal. Webbia, 62(2): 205-216.

Toulmin C, Guèye B. 2003. Transformation de l'agriculture Ouest africaine et rôle des exploitations familiales, dossier $\mathrm{N}^{\circ}$ 123.

Traoré H, Maillet J. 1992. Flore adventice des cultures céréalières annuelles du Burkina-Faso. Weed Research, 32: 279293.

Trochain JL. 1966. Types biologiques chez les végétaux intertropicaux (Angiosper- mes). Bull. Soc. Bot. de France; 188196.

Vanden Berghen C. 1982. Initiation à l'Etude de la Végétation ( ${ }^{\mathrm{e}}$ edn). Jardin Botanique National de Belgique.

Vanden Berghen C. 1998. Irradiations de la flore sahélienne en Basse Casamance (Sénégal). In : Atelier sur flore, végétation et biodiversité au Sahel, Bâ AT, Madsen JE, Sambou B (eds). AAU Report, 39, pp 151-161.

Zoundi SJ, Léonidas H. 2003 : Défis de l'accès des exploitations familiales aux innovations agricoles en Afrique de l'Ouest : implications institutionnelles et politiques. Club du Sahel et de L'Afrique de l'Ouest, OCDE, Paris. 